\title{
Mehrsprachigkeit und Schulerfolg - die europäische (deutsche) Perspektive
}

\author{
Claus Altmayer \\ Universität Leipzig, Herder-Institut, Beethovenstraße 15, D-04107 Leipzig \\ E-mail: altmayer@rz.uni-leipzig.de
}

\begin{abstract}
Multilingualism and multilingual education in Africa are different from multilingualism and multilingual education in Europe. Nevertheless, to some extent the circumstances are comparable. In both regions there are currently important academic and political discussions about the significance of the first language in the process of second language acquisition and about the effectiveness of bilingual education. Against this background the paper outlines the discussions in Germany about the impact "mother tongue education" may have on the acquisition of a second language (German), on the one hand, and on the different models of bilingual education, on the other hand, especially with regard to migrant contexts. There is some research on these topics, but more empirical evidence is urgently needed, in Europe as well as Africa.
\end{abstract}

Keywords: multiligualism, migration, mother tongue education, language of origin, bilingual education, interdependece hypothesis, conceptual writing

Schlüsselbegriffe: Mehrsprachigkeit, Migration, Muttersprache-unterricht, Herkunftssprache, bilingualer Unterricht, Interdependenzhypothese, konzeptionelle Schriftlichkeit

\section{Einleitung: Mehrsprachigkeit in Afrika und Europa - Gemeinsamkeiten und Unterschiede}

Mehrsprachigkeit ist heute nicht nur in Afrika, sondern zunehmend auch in Europa zu einer großen Herausforderung für die Gesellschaft, die Wirtschaft und insbesondere die Bildungssysteme geworden. Allerdings sind die Bedingungen in vielen Ländern Afrikas auf der einen und in Europa auf der anderen Seite natürlich nach wie vor sehr unterschiedlich. Während Europa mit dem aufkommenden Nationalismus im 19. Jahrhundert die bis dahin übliche territoriale Mehrsprachigkeit, d.h. die Existenz mehrerer unterschiedlicher Sprachen auf einem nationalen bzw. staatlichen Territorium, vergleichsweise erfolgreich bekämpft und das bis heute wirksame Prinzip ,Ein Land - eine Sprache' durch entsprechende Maßnahmen gegenüber Minderheitensprachen vielfach gewaltsam durchgesetzt hat, ist dies in Afrika erkennbar nicht der Fall. Hier existieren auf einem nationalstaatlichen Territorium bekanntlich häufig sehr viele und teilweise sehr verschiedene Sprachen nebeneinander, von denen nicht wenige über mehrere Millionen, auf bis zu 15 verschiedene Staaten verteilte Sprecher verfügen, von denen aber viele auch nur sehr kleine Sprachgemeinschaften umfassen und keinerlei offiziellen Status genießen. Die territoriale Mehrsprachigkeit geht hier in aller Regel 
mit einer ausgeprägten individuellen Mehrsprachigkeit einher, d.h. fast alle Menschen verfügen über mehr oder weniger entwickelte Kompetenzen in mehr als einer, nicht selten auch in vier oder fünf verschiedenen Sprachen. Hinzu kommt die Existenz der europäischen Kolonialsprachen Englisch, Französisch und Portugiesisch, die in den ehemaligen Kolonien des westlichen, östlichen und südlichen Afrika als Verkehrssprachen dienen.

Mehrsprachigkeit ist in Afrika nicht nur, aber zweifellos auch und vor allem ein Problem der Bildungssysteme. Der Zugang zu Bildung, insbesondere zu höherer Bildung und damit auch zu höheren gesellschaftlichen Positionen ist nach wie vor in hohem Maß von der Verfügung über ausreichende Kompetenzen in den europäischen Kolonialsprachen Englisch, Französisch oder Portugiesisch abhängig, denn schulische Bildung findet insbesondere in den ehemaligen französischen Kolonien des westlichen Afrika bis heute nahezu ausnahmslos in französischer Sprache statt, und auch in den ehemals britischen oder portugiesischen Kolonien ist die Situation nicht prinzipiell anders. Da aber die meisten Kinder zu Beginn des Schulbesuchs nicht über ausreichende Kenntnisse in diesen Sprachen verfügen und da die Schulen, insbesondere im frankophonen Afrika, auch keine entsprechenden Angebote machen, führt dies zu einer geradezu exorbitant hohen Abbrecherquote von teilweise bis zu $83 \%$ (vgl. Bamgbose 2000:84). In letzter Zeit findet daher die von der UNESCO bereits in den 50er Jahren formulierte Forderung nach einem Unterricht, der in der Erstsprache der Kinder (,mother tongue education') angeboten wird und bei dem die europäischen Sprachen zwar Gegenstand des Lernens, aber nicht Medium des Unterrichts sind, in vielen afrikanischen Ländern auch auf staatlicher Seite immer mehr Unterstützung, allerdings bestehen nach wie vor erhebliche Probleme und Unklarheiten bei der konkreten Realisierung. Diskutiert wird u.a. darüber, wie lange der Unterricht ausschließlich in der L1 der Schüler stattfinden soll, ab wann und in welcher Form die L2 (also in der Regel Englisch oder Französisch) einbezogen werden soll oder welche Modelle bilingualer Erziehung am ehesten Erfolg versprechend sind (vgl. Alidou et al. 2006:56ff.).

Die Diskussion über Mother Tongue Education ist in vielerlei Hinsicht ein spezifisch afrikanisches Phänomen, das in der (post)kolonialen Geschichte des Kontinents begründet ist. Gegenüber vorschnellen Vergleichen und Parallelisierungen mit Debatten, die anderswo unter gänzlich anderen Vorzeichen geführt werden, ist daher sicherlich Vorsicht geboten. Gleichwohl könnte es für beide Seiten, die afrikanische wie die europäische, interessant sein, die afrikanische Diskussion über Mother Tongue Education mit Diskussionen über herkunftssprachlichen oder bilingualen Unterricht in Verbindung $\mathrm{zu}$ bringen, wie sie in Europa im Zusammenhang mit der dort in den letzten Jahren entstandenen migrationsbedingten Mehrsprachigkeit geführt werden.

Der folgende Beitrag möchte eine solche Verbindung herstellen, indem er die Diskussion über den Zusammenhang zwischen (migrationsbedingter) Mehrsprachigkeit und Schulerfolg, wie sie derzeit in Deutschland als einem der großen europäischen Ländern geführt wird, kurz skizziert und bewertet. Der Fokus auf Deutschland ist dabei zum einen in der fachlichen Zuständigkeit und Kompetenz des Autors, zum anderen aber auch darin begründet, dass sich am Beispiel Deutschland einige grundlegende Probleme aufzeigen lassen, mit denen Europa insgesamt derzeit zu tun hat. Vieles spricht ja dafür, dass Europa für den Umgang mit Mehrsprachigkeit von Afrika einiges lernen kann, verfügt dieser Kontinent doch über ungleich mehr Erfahrung in dieser Hinsicht als das nach wie vor dominant monolinguale Europa. 


\section{Migrationsbedingte Mehrsprachigkeit in Deutschland}

Wenn in Deutschland und Europa von Mehrsprachigkeit die Rede ist, sind zwei verschiedene und sich nur teilweise überlappende Kontexte zu unterscheiden: Da ist zum einen der Kontext der europäischen (Fremd-)Sprachenpolitik, die angesichts der real fast überall vorherrschenden Einsprachigkeit und der damit einher gehenden Defizite an gesamteuropäischer Verständigung die Mehrsprachigkeit zur übergeordneten Zielsetzung gemacht hat. Jeder Bürger der Europäischen Union sollte zukünftig über seine Muttersprache hinaus (mindestens) zwei weitere europäische Sprachen sprechen; von diesen Fragen soll im Folgenden nicht oder nur am Rande die Rede sein. Zum anderen nämlich ist Mehrsprachigkeit in Europa in den letzten Jahren und Jahrzehnten auch als eine reale Erfahrung relevant geworden, die das traditionelle Prinzip der territorialen Einsprachigkeit zunehmend in Frage stellt und für die Bildungs- und Schulpolitik insbesondere in Mittel-, West- und Nordeuropa eine Herausforderung darstellt. Gemeint ist die durch die anhaltende Migration entstandene Mehrsprachigkeit, die in Deutschland vor allem in den westlichen (,alten') Bundesländern und hier wiederum vor allem in den großen Städten zur Normalität geworden ist.

Zwei neuere Erhebungen zur Mehrsprachigkeit an deutschen Schulen, die in Essen und Hamburg durchgeführt wurden, haben diese Normalität einer migrationsbedingten Mehrsprachigkeit deutlich sichtbar gemacht. Demnach sprechen zwar weiterhin über $70 \%$ der Grundschüler nur Deutsch, aber immerhin schon fast 30\% geben an, zu Hause neben Deutsch noch eine weitere Sprache zu sprechen. Dabei ist auch die Vielfalt der vertretenen Sprachen erstaunlich. Am häufigsten vertreten ist sowohl in Essen als auch in Hamburg das Türkische, daneben spielen Polnisch, Russisch, Arabisch eine große, Englisch, Serbisch/Kroatisch/Bosnisch, Farsi, Kurdisch sowie 70-90 weitere Sprachen eine kleinere Rolle (vgl. Chlosta/Ostermann 2008:19ff.). Wichtig an diesen Erhebungen ist, dass sie - anders als die amtlichen Statistiken, die in der Regel nur zwischen ,ausländischen' und ,deutschen' Schülern unterscheiden - das Problem nicht an der Staatsangehörigkeit der Kinder festmachen, sondern die tatsächliche Sprachensituation unabhängig von den rechtlichen Statusfragen erfassen. Das ist insofern relevant, als viele in Deutschland lebende Menschen zwar einen deutschen Pass haben, in ihren Familien aber gleichwohl nicht (nur) Deutsch, sondern eine andere Sprache sprechen, weil sie entweder eingebürgert oder als ,Aussiedler' aus Russland oder Polen eingewandert sind und somit als Deutsche gelten. Wir sprechen daher im Kontext der Bildungs- und Schulpolitik nicht mehr von ,deutschen' und , ausländischen' Kindern, sondern lieber von Kindern mit bzw. ohne ,Migrationshintergrund', weil dieser Begriff die Migrations- und damit auch die Sprachensituation der betreffenden Familien eher erfassen kann. Im Übrigen hat sich das Statistische Bundesamt seit 2005 dieser Sprachregelung angepasst und erfasst seitdem ebenfalls nicht mehr nur die Staatsangehörigkeit, sondern eben auch den Migrationshintergrund.

Auf die Details der Migrationsgeschichte und der aktuellen Situation verschiedener Migrantengruppen in Deutschland kann ich aus Platzgründen hier nicht eingehen. Wichtig für unseren Zusammenhang ist aber, dass die Bundesregierung die Existenz einer Einwanderungssituation in Deutschland offiziell erst vor ca. zehn Jahren anerkannt hat und auch erst seitdem eine aktive Migrations- und Integrationspolitik betreibt. Dabei steht, bedingt vor allem durch den so genannten PISA-Schock, das Problem der sprachlichen Integration, d.h. des Deutsch als Zweitsprache-Erwerbs bei Kindern und Jugendlichen mit 
Migrationshintergrund, deutlich im Vordergrund. Die 2001 veröffentlichte internationale Bildungsvergleichsstudie PISA (Programme for International Student Assessment) hatte gezeigt, dass die Leistungen deutscher Schüler in den Bereichen Lesekompetenz, mathematische Kompetenz und naturwissenschaftliche Grundbildung teilweise deutlich unterhalb des internationalen Durchschnitts liegen und dass dies unter anderem mit der vergleichsweise gering ausgeprägten Bildungsbeteiligung von Familien mit Migrationshintergrund und mit mangelnden Sprachkenntnissen der Kinder aus Zuwandererfamilien zu tun habe. „Fast 50 Prozent der Jugendlichen aus Zuwandererfamilien überschreiten im Lesen nicht die elementare Kompetenzstufe 1, obwohl über 70 Prozent von ihnen die deutsche Schule vollständig durchlaufen haben“ (Artelt u.a. 2001:39). Darüber hinaus wirken sich die sprachlichen Defizite auch in den Sachfächern aus, ,,sodass Personen mit unzureichendem Leseverständnis in allen akademischen Bereichen in ihrem Kompetenzerwerb beeinträchtigt sind“ (ebd.). Die Einsicht, dass dieses ernüchternde Resultat nicht primär, wie manche Politiker glauben machen wollten, den Zuwandererfamilien selbst anzulasten ist, sondern in allererster Linie das Versagen der deutschen Bildungs- und Integrationspolitik dokumentiert, hat sich mittlerweile weitgehend durchgesetzt und $\mathrm{zu}$ vielfältigen Maßnahmen einer Förderung des Spracherwerbs bei Zuwandererkindern geführt. Dabei wird die Notwendigkeit, die teilweise erheblichen Mängel vieler Kinder auch noch der zweiten und dritten Generation der Zuwandererfamilien im Deutschen zu beseitigen und den Kindern zu einer den schulischen Anforderungen entsprechenden Kompetenz im Deutschen zu verhelfen, von allen Beteiligten anerkannt; hochgradig umstritten ist aber, ob bzw. inwieweit dies auf Kosten der Familiensprachen und der Mehrsprachigkeit der Kinder gehen soll, welche Bedeutung die Mehrsprachigkeit zugewanderter Kinder überhaupt hat und wie sie sich auf deren Schulerfolg auswirkt. Dabei lassen sich mehrere Teilaspekte dieser Diskussion unterscheiden, von denen im Folgenden zwei etwas näher beleuchtet werden sollen: die Rolle, die den Herkunftssprachen der Kinder zugesprochen wird, sowie die Wirkungsweise verschiedener Modelle bilingualer Erziehung.

\section{Die Rolle der Herkunftssprachen}

Als ,Herkunftssprachen' (auch ,Familiensprachen') werden in der deutschsprachigen Diskussion die Sprachen bezeichnet, die Zuwanderer aus ihren Herkunftsregionen mitbringen, die meist in privat-familiären Kontexten weiterhin verwendet und an die nachwachsenden Generationen weitergegeben werden. Die Diskussion über die Frage, welche Bedeutung diesen Sprachen im Rahmen einer staatlichen Migrationspolitik zukommt und inwieweit ihre Förderung eine Aufgabe der staatlichen (deutschen) Bildungsbürokratie ist, durchzieht die Migrationsgeschichte in Deutschland seit ihren Anfängen und ist gerade in allerjüngster Zeit wieder hochaktuell geworden. Dass sich an diesem Punkt die Geister scheiden, zeigt allein schon die Tatsache, dass die für die schulische Bildung zuständigen Bundesländer sich von Anfang an nicht auf eine gemeinsame Linie einigen konnten, so dass nur ein Teil der Bundesländer (u.a. Bayern, Hessen, Nordrhein-Westfalen, Sachsen) den so genannten ,muttersprachlichen Ergänzungsunterricht' in bis zu 19 verschiedenen Sprachen als offizielles, wenn auch freiwilliges Zusatzangebot der Schulen eingeführt hat, während andere Länder (u.a. Baden-Württemberg, Saarland, Schleswig-Holstein) dies den Konsulaten der Herkunftsländer überlässt (vgl. Reich 2008:451ff.). Dabei orientierte sich der Herkunftssprachenunterricht sowohl als muttersprachlicher Ergänzungsunterricht der Schulen als auch als Angebot der Konsulate häufig an dem (nicht immer offen ausgesprochenen) Ziel, mit der Förderung der Herkunftssprachen bei nachwachsenden Generationen von 
Zuwanderern deren Option für eine Rückkehr in die ehemaligen Herkunftsländer offen zu halten - eine Option, die zu keinem Zeitpunkt realistisch war und die auch nur in wenigen Fällen (z.B. bei vielen griechischen Zuwanderern) realisiert wurde.

Mit den geänderten Prioritäten der deutschen Zuwanderungspolitik seit der Jahrhundertwende und dem neuen Akzent auf Integration ist in allerjüngster Zeit wieder eine Diskussion über den Sinn des Herkunftssprachenunterrichts entbrannt, die sich vor allem um die Frage dreht, ob dieser Unterricht und die dadurch ja explizit geförderte Mehrsprachigkeit von Kindern mit Migrationshintergrund zu deren Integration in die deutsche Gesellschaft beiträgt oder ob diese dadurch nicht eher behindert wird. Dabei wird von den Gegnern des Herkunftssprachenunterrichts zunächst darauf hingewiesen, dass es bislang keine belastbaren wissenschaftlichen Studien gebe, die eine positive Wirkung der Mehrsprachigkeit und insbesondere einer herkunftssprachlichen Kompetenz bei Kindern mit Migrationshintergrund belegen könnten, im Gegenteil: Auf der Basis von lerntheoretischen Prinzipien, wonach das Erlernen bestimmter Inhalte und Kompetenzen vor allem von der Zeit abhänge, die man dafür aufwende (time-on-task-Theorie), sei - so der Erziehungswissenschaftler Diether Hopf - zu erwarten, dass die Beschäftigung mit den Herkunftssprachen zu Lasten der Beschäftigung mit der Landessprache Deutsch gehe und sich daher negativ auf den Erwerb des Deutschen und damit auf den Prozess der Integration auswirke (vgl. Hopf 2005). Ähnlich, allerdings auf einer ungleich breiteren Datenbasis, argumentiert auch der Soziologie Hartmut Esser, der die Debatte über den Herkunftssprachenunterricht in letzter Zeit stark dominiert hat: Entscheidend sowohl für den Schulerfolg von Migrantenkindern als auch für den Erfolg von Migranten auf dem deutschen Arbeitsmarkt seien vor allem Kompetenzen in der Landessprache Deutsch. Dagegen lasse sich ein signifikanter Zusammenhang zwischen kompetenter Mehrsprachigkeit und damit auch dem Verfügen über eine der großen Herkunftssprachen von Migranten in Deutschland (z.B. Türkisch, Italienisch, Russisch usw.) auf der einen und einer erfolgreichen Bildungs- und Berufskarriere auf der anderen Seite in den vorliegenden empirischen Daten nicht nachweisen. Mehrsprachigkeit und ethnische Ressourcen seien für die strukturelle und soziale Integration von Migranten in Deutschland mehr oder weniger irrelevant (vgl. Esser 2006a: 544f.; Esser 2006b: 100f.).

Eine wichtige, wenn nicht entscheidende Rolle bei der Diskussion über die Bedeutung der Herkunftssprachen spielt bis heute die von Jim Cummins in den 70er Jahren des vergangenen Jahrhunderts entwickelte Interdependenzhypothese, wonach sich eine höhere, d.h. über die mündliche Alltagssprache hinausgehende schriftsprachliche Kompetenz in der L1 positiv auf die entsprechenden Kompetenzen in der L2 auswirke, dass demnach die intensivere Beschäftigung mit der L1 über die damit einhergehende Erweiterung der L2-Kompetenzen sehr wohl einen positiven Effekt auf den Schulerfolg von Migrantenkindern ausübe (vgl. Cummins 1979). Die Interdependenzhypothese wird daher von den Befürwortern des herkunftssprachlichen Unterrichts gerne als Pro-Argument angeführt, zumal neuere Untersuchungen den von Cummins eher theoretisch als empirisch hergestellten Zusammenhang zu bestätigen scheinen. So haben Baur/Meder schon Ende der 80er Jahre des 20. Jahrhunderts auf der Grundlage von Daten von 600 türkisch- und jugoslawischstämmigen Schülerinnen und Schülern festgestellt, dass in der Tat eine signifikante statistische Korrelation zwischen den Kompetenzen in der jeweiligen L1 (türkisch bzw. serbisch/kroatisch) und den Kompetenzen in der L2 Deutsch besteht (vgl. Baur/Meder 1991). Und die vor allem in Deutschland entstandene so genannte DESI-Studie, die nach den Kompetenzen 15jähriger Schüler in Deutsch und Englisch fragte, konnte eine positive 
Korrelation zwischen mehrsprachiger Kompetenz von Schülern und deren Leistungen in der Fremdsprache Englisch feststellen (vgl. DESI-Konsortium 2006:22ff.), eine Korrelation, die im Allgemeinen damit erklärt wird, dass mehrsprachige Schüler über ein höheres Maß an Sprachlernerfahrung verfügen, die sich auf den Erwerb jeder weiteren Sprache positiv auswirkt.

Die Diskussion über die Rolle der Herkunftssprachen im Hinblick auf den schulischen Erfolg und den Erwerb der Zweitsprache Deutsch bei Kindern mit Migrationshintergrund ist nicht abgeschlossen. Insbesondere die Gültigkeit der Interdependenzhypothese ist nach wie vor umstritten, denn es ist keineswegs ausgemacht, ob die z.B. von Baur/Meder festegestellten Korrelationen tatsächlich auf einen kausalen Zusammenhang zwischen L1-Kompetenz und L2-Erwerb zurückgeführt werden können oder ob nicht diesem Zusammenhang eine dritte, nicht gemessene Variable zugrunde liegt (vgl. Esser 2006b:63); hier besteht zweifellos noch weiterer Forschungsbedarf in erheblichem Umfang. Gleichwohl hat die Diskussion über die Bedeutung der Herkunftssprachen für den Erwerb der Zweitsprache Deutsch in Migrationskontexten bereits zu begrifflichen und theoretischen Differenzierungen geführt, die auch und gerade für den afrikanischen Kontext von Mehrsprachigkeit interessant und relevant sein könnten. So gehen wir beispielsweise heute (und in Anknüpfung an Cummins) davon aus, dass wir beim Begriff der ,Sprachkompetenz' zwischen einem privat-familiären Alltagskontext einerseits und einem formellen Schulkontext andererseits unterscheiden und für letzteren insbesondere eine spezifische Sprachkompetenz definieren müssen, die bei Cummins , cognitive academic language proficiency' (CALP) hieß, für die sich aber mittlerweile im Fach Deutsch als Fremd- und Zweitsprache der präzisere Begriff der ,konzeptionellen Schriftlichkeit' eingebürgert hat, eine spezifisch schulbezogene Fachsprache also, für die Merkmale wie Kontextunabhängigkeit, Monologizität, das Vorherrschen hypotaktischer Strukturen oder eine merkmalsreichere Lexik kennzeichnend sind (vgl. Kniffka/Siebert-Ott 2007:19 f.). Vieles scheint dafür zu sprechen, dass gerade im Bereich der konzeptionellen Schriftlichkeit die L2-Kompetenz in hohem Maße vom Vorhandensein oder Nichtvorhandensein einer entsprechenden L1-Kompetenz abhängt. Wer gelernt hat, seine L1 in konzeptioneller Schriftlichkeit kompetent zu verwenden, wird diese Kompetenz eher auf eine entsprechende Anforderung in der L2 übertragen können als derjenige, der schon in der L1 nicht über derartige Fähigkeiten verfügt. Wie sich dieser Zusammenhang konkret und im Einzelnen darstellt, ist aber bisher noch nicht in ausreichendem Maß erforscht.

\section{Modelle bilingualer Erziehung}

Das deutsche Schulsystem war auf den sich spätestens seit den 70er Jahren des 20. Jahrhunderts deutlich verstärkenden Zulauf von Kindern mit Migrationshintergrund, die teilweise über keine oder nicht ausreichende Kenntnisse in der Schulsprache Deutsch verfügten, nicht vorbereitet und reagierte mit einer Vielzahl unterschiedlicher Konzepte und Fördermaßnahmen, die von der reinen Submersion über spezielle Vorbereitungsklassen bis hin zu differenzierten Modellen einer allmählichen Integration in den Regelunterricht reichen (vgl. zu diesen älteren Konzepten Beauftragte der Bundesregierung für Ausländerfragen 2001). Hinzu kommen in letzter Zeit vor allem Konzepte einer Frühförderung der Kinder in der Zweitsprache Deutsch, die bereits in der Schuleingangsphase, also vor Beginn des eigentlichen Schulbesuchs, ansetzen und zum Ziel haben, die Kinder so weit in der Schulsprache Deutsch zu fördern, dass sie den sprachlichen Anforderungen der Schule von Anfang an gewachsen sind, bei denen aber die Herkunftssprachen der Kinder keinerlei 
Berücksichtigung finden. Insgesamt ist derzeit im öffentlichen Diskurs die Tendenz festzustellen, die zeitweise stärker anerkannte reale Mehrsprachigkeit an deutschen Schulen wieder zurück zu drängen und nur noch die Kompetenzen in der Zweitsprache Deutsch zu fördern. So wird beispielsweise in einigen Bundesländern erwogen, den muttersprachlichen Ergänzungsunterricht abzuschaffen bzw. auch dort, wo dies bislang nicht der Fall war, in die Verantwortung der Konsulate der Herkunftsländer zu übertragen; in Hessen und Bayern ist dies bereits beschlossene Sache (vgl. Reich 2008:452).

Andererseits sehen auch die Befürworter des herkunftssprachlichen Unterrichts in den seit vielen Jahren praktizierten Programmen auch nicht unbedingt die Lösung des Problems, da sowohl der von den Bildungsbehörden der Bundesländer verantwortete muttersprachliche Ergänzungsunterricht als auch die Angebote der Konsulate nicht in den Regelunterricht integriert und somit auch nicht mit dem Unterricht in der Zweitsprache Deutsch koordiniert sind. Favorisiert werden daher meist Modelle einer bilingualen Erziehung, bei denen man sich an den international üblichen, vor allem in Kanada und den USA praktizierten Konzepten orientiert, bei denen man in Deutschland bisher aber über vergleichsweise wenig Erfahrung verfügt. Zwar gibt es mittlerweile eine steigende Zahl von Gymnasien, die bilinguale Angebote machen, dabei geht es aber in der Regel um prestigeträchtige Weltsprachen wie insbesondere das Englische und zudem um Angebote, die sich vorrangig an die Angehörigen der Mehrheitsgesellschaft (und hier wiederum vor allem mittlerer und höherer sozialer Schichten) wendet und nicht an Kinder mit Migrationshintergrund. Ebenfalls für unsere Fragestellung weniger relevant sind bilinguale Modelle in den (wenigen) autochthonen Minderheitensprachen wie Sorbisch in Sachsen und Brandenburg oder Dänisch und Friesisch in Schleswig-Holstein, die ja vor allem den Erhalt dieser bedrohten Minderheitensprachen zum Ziel haben. Bilinguale Modelle, die das Deutsche mit einer der Herkunftssprachen wie Türkisch, Russisch oder Polnisch kombinieren, sind nach wie vor höchst selten und haben zudem durchweg den Status von Versuchs- oder Modellschulen, wobei sich die konkrete Ausgestaltung des bilingualen Unterrichts je nach Schule und Bundesland unterscheidet. Immer wieder herangezogene Beispiele für bilinguale Modelle mit Einbeziehung der Herkunftssprachen von Migrantenkindern sind etwa die Staatlichen Europaschulen in Berlin, die Deutsch-Italienische Schule in Wolfsburg oder die bilinguale Grundschule in Hamburg (vgl. dazu u.a. Gogolin 2005).

Die wissenschaftliche Diskussion über die Effizienz bilingualer Modelle ist in Deutschland bisher eher unterentwickelt und orientiert sich stark an dem, was anderswo, speziell in den USA und in Kanada, an Erfahrungen, an empirischen Daten und an wissenschaftlichen Auswertungen vorliegt. Bei der Frage, ob die bilinguale Erziehung tatsächlich, wie von ihren Befürwortern behauptet, nicht nur Mehrsprachigkeit allgemein fördert, sondern insbesondere $\mathrm{zu}$ besseren Leistungen in der Zweitsprache und damit zu besseren Schulerfolgen beiträgt, stützt man sich daher auch in Deutschland meist auf die Studien, die dazu aus den USA vorliegen, die auch anderswo - auch in Afrika - häufig herangezogen werden und die in der Regel die Überlegenheit bilingualer gegenüber monolingualen Programmen konstatieren: die Studien von Ramirez et al. (1991a, 1991b, 1991c) und von Thomas/Collier (1997, 2002) sowie die Metaanalysen von Greene (1998) und von Slavin/Cheung (2005). Neuere Metaanalysen, die teilweise auch in Deutschland entstanden sind (vgl. Söhn 2005, Esser 2006a:387ff.; Rossell/Kuder 2005), sehen dies jedoch wieder deutlich kritischer. Auf der Basis einer umfangreichen kritischen Überprüfung der vorliegenden Studien und Metaanalysen und der ihnen zugrunde liegenden methodischen Vorgaben kommt 
beispielsweise Janina Söhn in ihrer Analyse zu dem Ergebnis, dass sich die bilinguale Erziehung zwar einerseits nicht negativ auf den Erwerb der Zweitsprache auswirke, dass aber andererseits auf der Grundlage der bislang vorliegenden Daten auch ein positiver Effekt nicht nachweisbar sei (vgl. Söhn 2005:64). Um Missverständnisse zu vermeiden, sei hier darauf hingewiesen, dass dieses Resultat sich vor allem aus den methodischen Mängeln der vorliegenden Studien ergibt und daher nicht umgekehrt als Nachweis einer Ineffizienz bilingualer Programme gelesen werden darf. Deutlich wird hier vor allem eines: Wir brauchen mehr und bessere Forschung zum Zusammenhang zwischen Mehrsprachigkeit und Schulerfolg im Allgemeinen und zur Wirksamkeit bilingualer Modelle im Besonderen.

\section{Fazit und Ausblick}

Es sollte anhand der hier kurz skizzierten Debatten deutlich geworden sein, dass Mehrsprachigkeit auch in Deutschland und Europa zu einem nicht nur punktuellen, sondern grundsätzlichen und strukturellen Problem für die traditionell monolingual orientierten Bildungssysteme geworden ist, von dessen nachhaltiger Lösung die weitere Entwicklung der Bildung und der Gesellschaft in hohem Maße abhängt. Es wurde zu Beginn bereits darauf hingewiesen, dass eine vorschnelle Verbindung zwischen dieser spezifisch deutschen bzw. europäischen Diskussion und der ebenso spezifisch afrikanischen Diskussion über die Mother Tongue Education sicherlich nicht angemessen wäre. Gleichwohl lassen sich auch interessante Parallelen nicht von der Hand weisen, die eine zukünftig genauere Beschäftigung mit diesen Zusammenhängen immerhin erstrebenswert erscheinen lassen. In Europa wie in Afrika geht es um die Frage, ob, in welchem Maß und in welcher Weise die Einbeziehung der Erstsprachen mehrsprachig aufwachsender Kinder in den Bildungssystemen deren Bildungskarrieren beeinflusst, indem die Nichtberücksichtigung dieser Sprachen zum Scheitern oder doch zumindest zu niederrangigen Bildungsabschlüssen führt. In beiden Fällen wird mit verschiedenen Modellen einer bilingualen Erziehung experimentiert, von denen bis jetzt nicht wirklich und zuverlässig gesagt werden kann, welche Effekte sie für die sprachliche Bildung und den davon abhängigen Schulerfolg tatsächlich haben. Und in beiden Fällen stützt man sich zudem auf wissenschaftliche Studien zum Zusammenhang von Mehrsprachigkeit und Schulerfolg, die vor allem in den USA entstanden sind, die schon aufgrund der völlig anderen sozialen und sprachlichen Bedingungen nicht ohne Weiteres auf die Verhältnisse in Afrika bzw. in Europa übertragen werden können und deren methodische Basis sich zudem in jüngster Zeit als doch reichlich problematisch herausgestellt hat. Weder im afrikanischen noch im europäischen Kontext gibt es bislang wissenschaftliche Expertise zu der uns interessierenden Frage in ausreichendem Maß. Hier gilt es in Zukunft anzusetzen, und hier sehe ich auch interessante Perspektiven für eine stärkere Zusammenarbeit zwischen Europa und Afrika, eine Zusammenarbeit, bei der Europa vor allem von den reichhaltigen afrikanischen Erfahrungen mit Mehrsprachigkeit viel wird lernen können.

\section{Bibliographie}

Alidou, H. et al. 2006. Optimizing learning and education in Africa - the language factor. A stock-taking research on mother tongue and bilingual education in sub-Saharan Africa. Paris: Adea. Online verfügbar unter http://www.adeanet.org/adeaPortal/adea/biennial2006/doc/document/B3_1_MTBLE_en.pdf; letzter Zugriff: 14.08.09.

Artelt, C. u.a. (Hg.) 2001. PISA 2000. Zusammenfassung zentraler Befunde. Berlin: Max Planck-Institut für Bildungsforschung. 
Bamgbose, A. 2000. Language and exclusion. The consequences of language policies in Africa. Münster etc.: Lit Verlag.

Baur, R.S. und G. Meder. 1991. Zur Interdependenz von Muttersprache und Zweitsprache bei jugoslawischen Migrantenkindern. In R. S. Baur u.a. (Hg.) Interkulturelle Erziehung und Zweisprachigkeit. Baltmannsweiler: Schneider. pp. 109-140.

Beauftragte der Bundesregierung für Ausländerfragen (Hg.) 2001. Mehrsprachigkeit an deutschen Schulen. Ein Länderüberblick. Berlin. Online verfügbar unter http://www.bundesregierung.de/Content/DE/Publikation/IB/Anlagen/mehrsprachigkeitan-schulen,property=publicationFile.pdf; letzter Zugriff 14.08.09.

Chlosta, C. und T. Ostermann. 2008. Grunddaten zur Mehrsprachigkeit im deutschen Bildungssystem. In B. Ahrenholz und I. Oomen-Welke (Hgg.) Deutsch als Zweitsprache. Baltmannsweiler: Schneider. pp. 17-30.

Cummins, J. 1979. Linguistic interdependence and the educational development of bilingual children. Review of Educational Research 49(2): 222-251.

DESI-Konsortium. 2006. Unterricht und Kompetenzerwerb in Deutsch und Englisch. Zentrale Befunde der Studie Deutsch-Englisch-Schülerleistungen-International (DESI). Frankfurt a.M.: DIPF.

Esser, H. 2006a. Sprache und Integration. Die sozialen Bedingungen und Folgen des Spracherwerbs von Migranten. Frankfurt a.M. und New York: Campus.

Esser, H. 2006b. Migration, Sprache und Integration. AKI-Forschungsbilanz 4. Berlin: Wissenschaftszentrum für Sozialforschung.

Gogolin, I. 2005. Bilingual education - the German experience and debate. In Arbeitsstelle Interkulturelle Konflikte und gesellschaftliche Integration (AKI) (Hg.) The effectiveness of bilingual school programs for immigrant children. Berlin: Wissenschaftszentrum Berlin für Sozialforschung. pp. 133-145.

Greene, J. P. 1998. A meta-analysis of the effectiveness of bilingual education. Los Angeles: University of Southern California, Tomas Rivera Policy Institute.

Hopf, D. 2005. Zweisprachigkeit und Schulleistung bei Migrantenkindern. Zeitschrift für Pädagogik 51(2): 236-251.

Kniffka, G. und G. Siebert-Ott. 2007. Deutsch als Zweitsprache. Lehren und lernen. Paderborn u.a.: Schöningh.

Ramirez, J.D. et al. 1991a. Longitudinal study of structured English immersion strategy, early-exit and late-exit transitional bilingual education programs for language-minority children. Final report - Executive summary. San Mateo: Aguirre International.

Ramirez, J.D. et al. 1991b. Longitudinal study of structured English immersion strategy, early-exit and late-exit transitional bilingual education programs for language-minority children. Final report - Volume I. San Mateo: Aguirre International.

Ramirez, J.D. et al. 1991c. Longitudinal study of structured English immersion strategy, early-exit and late-exit transitional bilingual education programs for language-minority children. Final report - Volume II. San Mateo: Aguirre International.

Reich, H.H. 2008. Herkunftssprachenunterricht. In B. Ahrenholz und I. Oomen-Welke (Hgg.) Deutsch als Zweitsprache. Baltmannsweiler: Schneider. pp. 445-456.

Rossell, C.H. und J. Kuder. 2005. Meta-murky: A rebuttal to recent meta-analyses of bilingual education. In Arbeitsstelle Interkulturelle Konflikte und gesellschaftliche Integration (AKI) (Hg.) The effectiveness of bilingual school programs for immigrant children. Berlin: Wissenschaftszentrum Berlin für Sozialforschung. pp. 43-76.

Slavin, R.E. und A. Cheung. 2005. A synthesis of research on language of reading instruction for English language learners. In Arbeitsstelle Interkulturelle Konflikte und 
gesellschaftliche Integration (AKI) (Hg.) The effectiveness of bilingual school programs for immigrant children. Berlin: Wissenschaftszentrum Berlin für Sozialforschung. pp. 542.

Söhn, J. 2005. Zweisprachiger Schulunterricht für Migrantenkinder. Ergebnisse der Evaluationsforschung zu seinen Auswirkungen auf Zweitspracherwerb und Schulerfolg. AKI-Forschungsbilanz 2. Berlin: Wissenschaftszentrum Berlin für Sozialforschung.

Thomas, W.P. und V. Collier. 1997. School effectiveness for language minority students. Washington: National Clearinghouse for Bilingual Education.

Thomas, W.P. und V. Collier. 2002. A national study of school effectiveness for language minority students. Long-term academic achievement. Santa Cruz, CA: Center for Research on Education, Diversity, and Excellence.

\section{Biographical note}

Claus Altmayer studied German and Philosophy at various universities in Germany. Ph.D. 1992 and Habilitation 2002, both from the University of Saarland at Saarbrücken. 1990 - 1994 DAAD Lecturer at the University of Latvia in Riga. 1994 - 2005 various appointments in German as a Foreign Language at the University of Saarland at Saarbrücken. Since 2005 Professor for German as a Foreign Language and Cultural Studies at the Herder-Institute of Leipzig University. 